\title{
Naphthalene Derivatives Induce Acyl Chain Interdigitation in Dipalmi- toylphosphatidylcholine Bilayers
}

\author{
Md.Arif Kamal and V.A Raghunathan
}

\section{Variation of the obliqueness of the unit cell of the ripple phase}

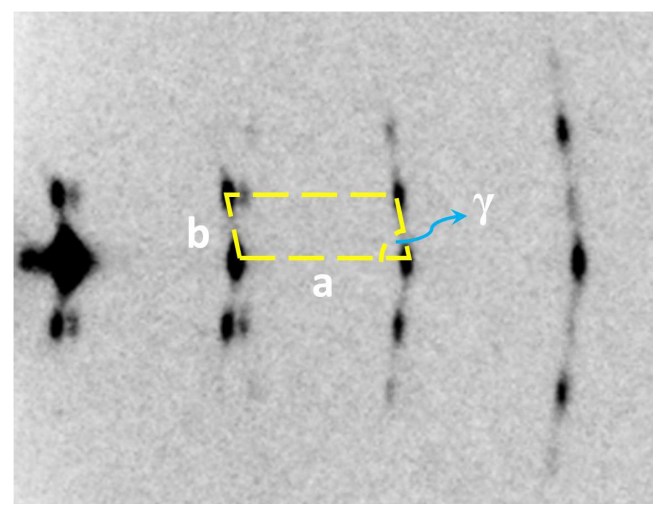

Figure S1 Lattice parameters of the ripplephase $\mathrm{P}_{\beta^{\prime}} . \xi$ is defined as $(\pi-\gamma)$
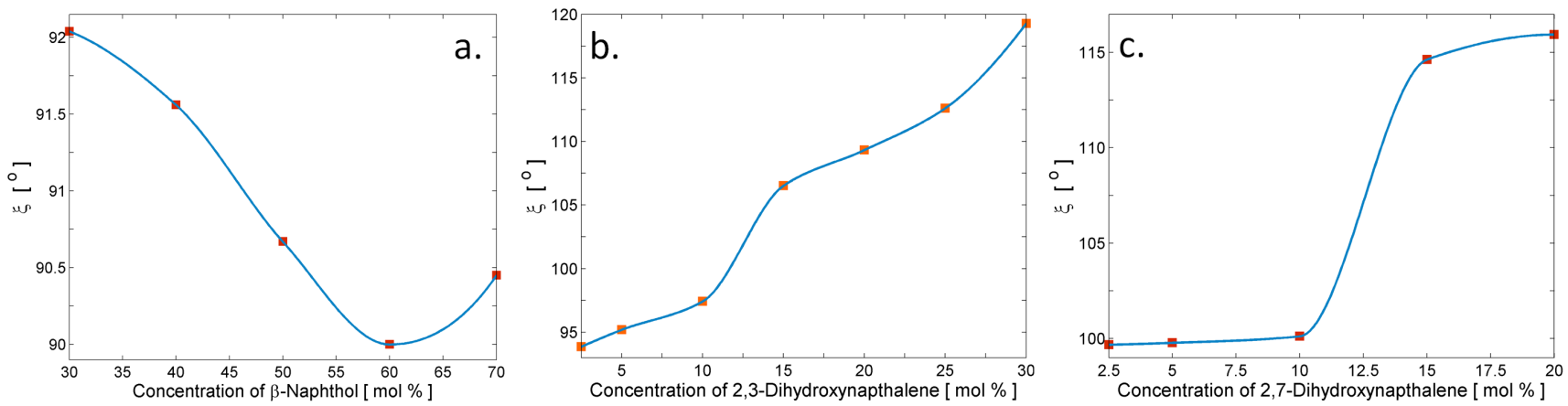

Figure S2 Variation of the lattice cell parameter $\xi$ of the ripple phase $\mathrm{P}_{\beta^{\prime}}$ at $25^{\circ} \mathrm{Cwith}$ increasing concentration of (a) $\beta$-naphthol, (b) 2,3-Dihydroxynaphthalene, and (c) 2,7-Dihydroxynaphthalene in the DPPC membrane. The typical error in $\lambda$ is $\pm 0.5 \AA$. The smooth lines are merely guides for the eye.

\section{Trans-bilayer Electron Density Profile}

More detailed structural information about the bilayer is contained in the trans-bilayer electron density profile $\rho(z)$, which can be calculated from the diffraction data. The Lorentz corrected scattered intensity from a stack of bilayers is given by ${ }^{1}$,

$$
I(q)=S(q)|f(q)|^{2}
$$

where $S(q)$ is the structure factor, and $f(q)$ the form factor. $f(q)$ is the Fourier transform of $\rho(z)$. In the simplest description, the membrane stack can be looked upon as a 1-D lattice of lamellar periodicity d, and then $\mathrm{S}(\mathrm{q})$ is a set of delta functions at $\mathrm{q}=h q_{0}$, with $q_{0}=2 \pi / d$ and $\mathrm{h}=1,2,3$, etc. The intensities of the set of lamellar reflections can then be written as

$$
I\left(h q_{0}\right)=\left|f\left(h q_{0}\right)\right|^{2}
$$

$\rho(z)$ can be calculated by Fourier inversion of $\mathrm{f}(\mathrm{q})$, if the phases of these reflections can be somehow determined. This problem is simplified by the fact that the phases of the reflections are constrained to be either 0 or $\pi$, since the flat bilayer is symmetric 


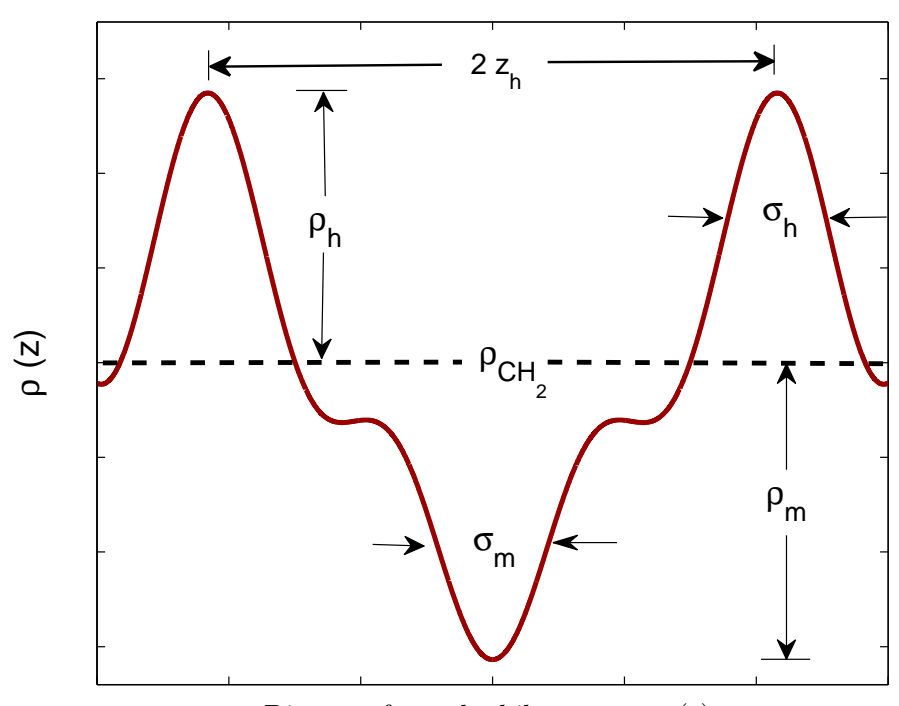

Distance from the bilayer centre (z)

Figure S3 3-Gaussian Model for the trans bilayer electron density profile.

about its mid-plane. With this simplification, for a bilayer-water system having h diffraction peaks the phase problem effectively boils down to finding the right combination of phases amongst the $2^{h}$ possibilities. Hence,

$$
\rho(z)=\sum_{h} \alpha_{h} f\left(h q_{0}\right) \cos \left(h q_{0} z\right)
$$

where $\alpha_{h}$ are the phases of different Fourier components $f\left(h q_{0}\right)$.

Various methods have been used to phase the reflections from the lamellar phases of bilayers. In one of them, a model for $\rho(z)$ is constructed based on some a priori knowledge of the structure of the phase. The unknown structural features are taken into account through a few adjustable parameters, whose values are determined from the best fit of the observed diffraction data with those calculated from the model. For example, $\rho(z)$ can be written as the sum of three Gaussian functions corresponding to the two electron-rich headgroups and the electron-deficient terminal methyl group (Fig. [S3]),

$$
\rho(z)=\rho_{h}\left\{e^{-\frac{\left(z+z_{h}\right)^{2}}{2 \sigma_{h}}}+e^{-\frac{\left(z-z_{h}\right)^{2}}{2 \sigma_{h}}}\right\}-\rho_{m} e^{-\frac{z^{2}}{2 \sigma_{m}}}
$$

where $\rho_{h}$ and $\sigma_{h}$ are the height and width of headgroup Gaussian function, and $\rho_{m}$ and $\sigma_{m}$ the corresponding parameters for the terminal methyl groups. The values of these parameters as well as that of $z_{h}$, which is related to the thickness of the bilayer, are obtained from the best fit of the observed values of the intensities with those calculated from the model. The phases of the reflections obtained from the model are then combined with their observed magnitudes and Fourier transformed to get the bilayer electron density profile $\rho(z)$. Several important structural parameters, such as the membrane thickness and number of water molecules per lipid, can be estimated once $\rho(z)$ is known ${ }^{1,2}$.

However, in systems where only 4 Bragg peaks are observed, it is easier employ the brute force method of testing all the $2^{4}$ possible combinations to get the right combination of phases which give a physically acceptable electron density profile. 
3 Plot of the bilayer thickness $d_{p p}$ and d-spacing as a function of 2,7-dihydroxynaphthalene concentration

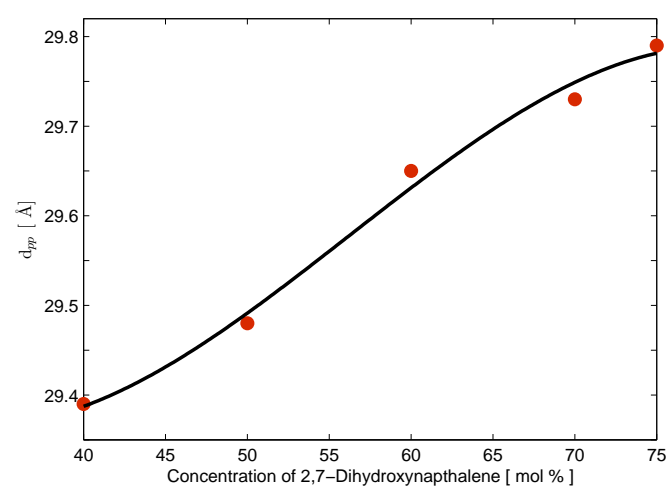

(a)

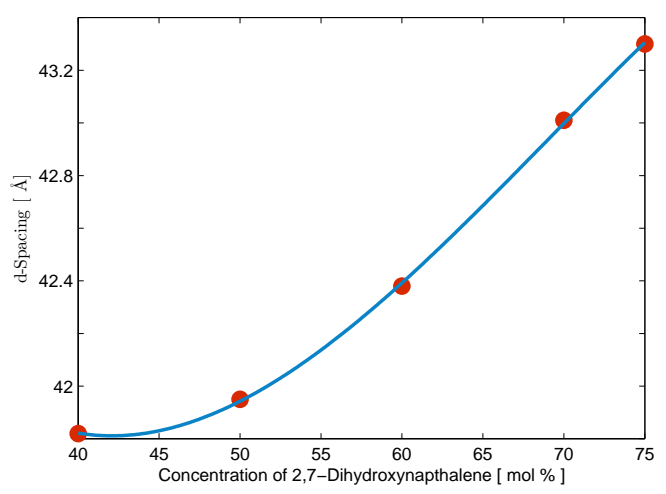

(b)

Figure S4 Plot of the (a) bilayer thickness $\mathrm{d}_{p p}$ and (b) d-Spacing as a function of 2,7-dihydroxynaphthalene concentration, in the $\mathrm{L}_{\beta I}$ phase at $10^{\circ} \mathrm{C}$. 
4 Comparison of Tran-bilayer Electron Density Profile (EDP) in $L_{\beta^{\prime}}$ and $L_{\beta I}$ phase.

\section{$4.1 \quad 2,3-d i h y d r o x y n a p h t h a l e n e$}

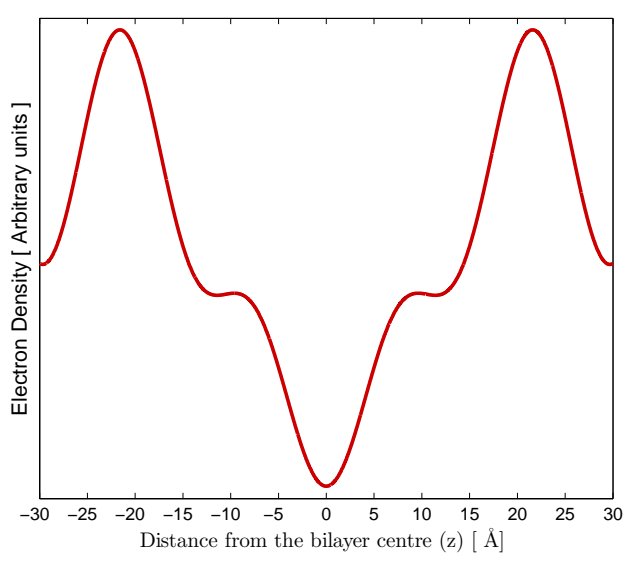

(a)

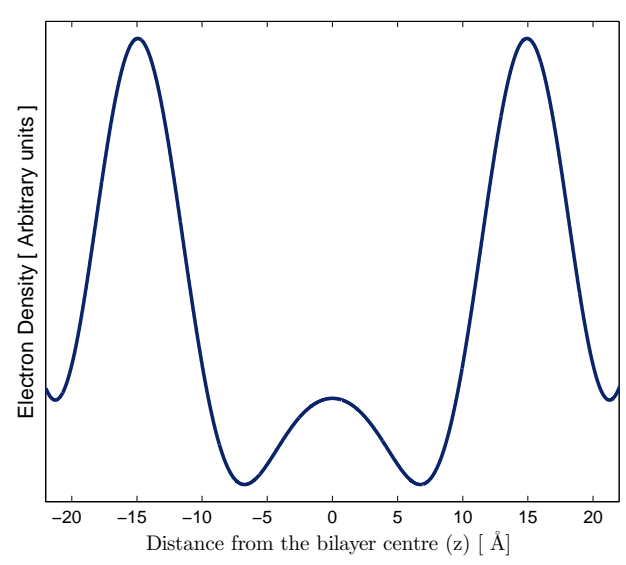

(b)

Figure S5 Plot of the transbilayer electron density profile in the (a) $\mathrm{L}_{\beta^{\prime}}$ phase at $1 \mathrm{~mol} \%$ and (b) $\mathrm{L}_{\beta I}$ phase at 60 mol $\%$ of 2,3-dihydroxynapthalene at $10^{\circ} \mathrm{C}$.

\subsection{2,7-dihydroxynaphthalene}

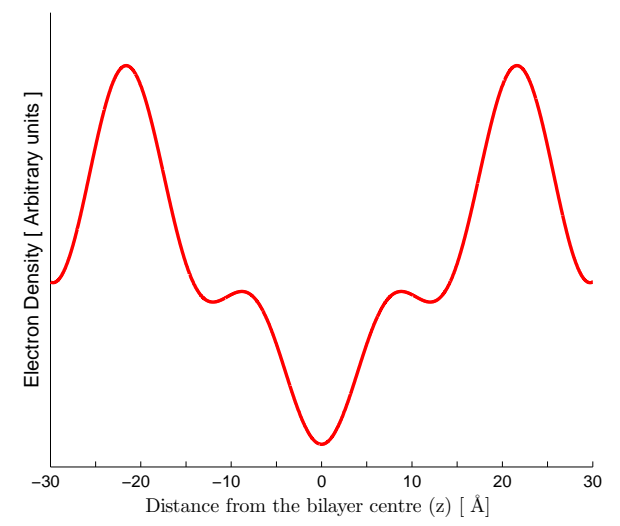

(a)

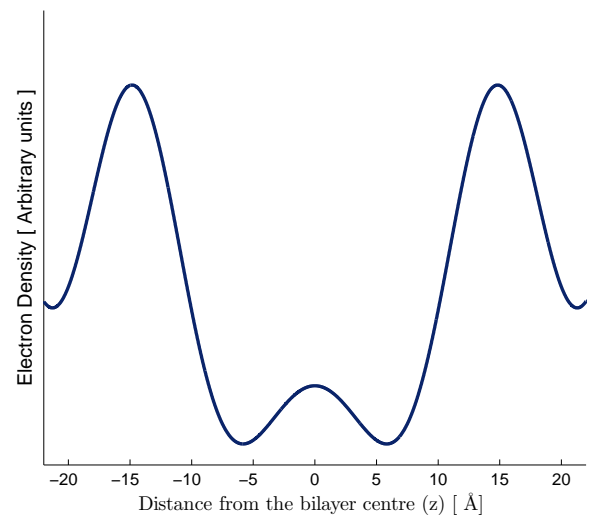

(b)

Figure S6 Plot of the trans-bilayer electron density profile in the (a) $\mathrm{L}_{\beta^{\prime}}$ phase at $1 \mathrm{~mol} \%$ and (b) $\mathrm{L}_{\beta I}$ phase at $60 \mathrm{~mol} \%$ of 2,7-dihydroxynapthalene at $10^{\circ} \mathrm{C}$.

\section{References}

[1] J. F. Nagle, and S. Tristram-Nagle, Biochim. Biophys. Acta, 1469, 159 (2000).

[2] J. F. Nagle, M. C. Wiener, Biophys. J., 55, 309 (1989). 